\title{
A MEGAVOLT TEST STAND FOR MEASURING CATHODE AND ANODE EMISSIONS WITH NANOSECOND PULSES *
}

\author{
R.J. Allen ${ }^{\xi}$, D.D. Hinshelwood, J.W. Schumer and I.M. Rittersdorf ${ }^{\text {a }}$ \\ Plasma Physics Division, Naval Research Laboratory \\ Washington, DC 20375 USA
}

\section{Abstract}

A new test stand has been constructed for measuring emissions from cathode and anode surfaces under high electrical stress in vacuum. An L-3 PulseRad is used as the generator to provide $\sim 50$-ns electrical pulses of up to 1 MV. The usual sealed glass $x$-ray tube has been replaced by a stacked ring insulator so that custom load hardware can be used with the generator [1].

The initial configuration of the load hardware was designed to measure electron emission from cathode surfaces. The cathodes are flat disks with a three inch OD. Using a flat cathode surface eliminates a geometric component of field enhancement. The anode sits below the cathode surface with an adjustable AK gap. With this geometry we can test areas of several square centimeters with variable peak electric fields up into the $\mathrm{MV} / \mathrm{cm}$ range.

A vacuum voltmeter is used in this configuration, both to carefully measure the applied voltage and to act as a dummy load. This eliminates concerns about damaging the generator in case the test load stays at high impedance. Bdot probes on the cathode holder measure the current from the sample. An x-ray camera placed above the system looks through the cathode at the high $\mathrm{Z}$ anode to give a time integrated view of $x$-ray emission from the anode, helping to diagnose uniformity of emission. Optical access to the cathode surface is provided by vacuum windows, allowing for the use of cameras, spectrometers and interferometers.

This new test stand will first be used to investigate the role of surface material, machining, cleaning and coatings on the temporal development of electron emission from cathode surfaces. Later, the test stand will be modified to examine ion emission from the cathode and anode surfaces.

\section{EMISSION PROCESSES IN HIGH- POWER, CROSS-FIELD VACUUM DEVICES}

At very high power levels, emission of particles into a vacuum gap from anode and cathode surfaces can occur. Usually, the first to occur and most dominant emission mechanism is explosive electron emission from the cathode. Explosive electron emission is believed to form from field enhanced sites on the cathode surface [2] that heat and explode from Joule heating due to high values of localized current, initially from electrons tunneling into vacuum. The site of the explosion becomes expanding plasma that becomes an emitter of electrons that is only space charge limited. Often, cathode surfaces are specially prepared to be either uniform emitters at low electric fields or non-emitting even at high electric field.

As the electron current from the cathode increases, the electron orbits can be bent by their self-magnetic field. Often, this results in areas of high current density on the anode which heats the anode surface. When the anode surface temperature is raised about $400{ }^{\circ} \mathrm{C}$ it is observed that a plasma forms on the anode surface which becomes a strong emitter of ions [3], a process called thermal ion emission.

Although explosive electron emission and thermal ion emission are often the two most dominant emission mechanisms, there are other emission processes that can sometimes play an important role in device behavior. Three of these are stimulated ion emission, negative ion emission and stimulated electron emission. Stimulated ion emission is believed to occur when electrons strike the anode with high energy but not enough current density to raise the temperature enough for thermal ion emission. There is believed to be a small but finite probability that an incident electron will liberate an ion from the anode surface. Negative ion emission has been measured from the plasma that forms on the cathode surface from explosive emission [4]. Stimulated electron emission can occur from non-emitting areas of the cathode surface when struck by an ion from the anode. A graphical representation of these five emission mechanisms is shown in Fig.1. Note that other mechanisms, such as field

\footnotetext{
* Work supported by the Naval Research Laboratory Base Program

$\xi$ email: allen@nrl.navy.mil

${ }^{a}$ National Research Council Research Associate
} 
desorption of ions from the anode may also have a role in device behavior in some circumstances.

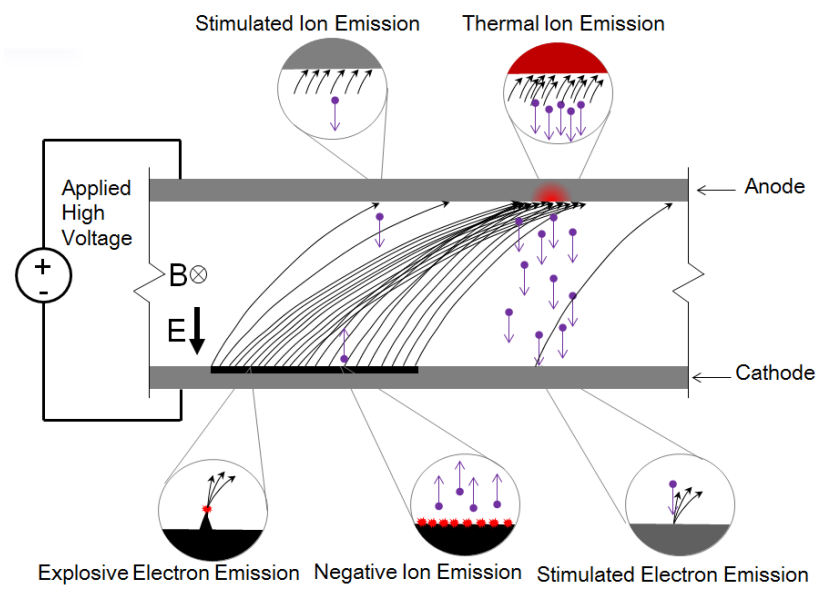

Figure 1. Graphical depiction of five emission processes in cross-field devices.

\section{EXPERIMENTAL SETUP}

In order to make measurements of these emission mechanisms, we have constructed a 1 MV test stand based on L-3 model 43710 S flash $\mathrm{x}$-ray machine rated for $1 \mathrm{MV}$ and $9 \mathrm{kA}$. Using this relatively small pulsed power generator allows us to acquire data at a high shot rate with minimal cost. The normal diode for this generator is a radiography diode inside a sealed glass tube that is surrounded by SF6. In earlier work, we have replaced this with a stacked ring insulator and a coaxial output with standard ISO100 tubing as the outer conductor [1]. For these measurements we have connected the ISO100 output to a six-way ISO100 cross where the anode and cathode are housed.

Although this setup will be used for measuring various forms of emission, we will describe the setup for the first tests, measuring explosive emission from a cathode. The cathodes are simple 3" OD disks, clamped in a holder and suspended by rods above the anode. The 3" cathode size was chosen because it could fit in our SEM without cutting. The simple geometry of the cathode allows testing of many samples with minimal expense.

The top port of the six-way cross is used to hold the cathode and also allows a view of the anode (through the cathode) for an x-ray pinhole camera. A bdot probe on the top port flange is used to measure cathode current, as shown in Fig. 2. The anode is a 1" diameter disk suspended from below by a vacuum voltmeter (VVM) and from the side by the center conductor of the generator's coaxial output. The VVM serves two purposes. In addition to directly measuring the voltage between anode and cathode, it serves as a matched load for the generator. This allows us to operate the generator at full voltage without risking damage in case the AK gap does not break down. The remaining three ports of the cross provide optical access for additional diagnostics. A photograph of this setup is shown in Fig. 3. A fourchannel gated optical imaging system (GOI) has recently been added to the setup. It is the black assembly on the left side of the setup in Fig. 3. The GOI allows us to take ns gated images of optical emission from the cathode, useful for localizing emission and observing uniformity.

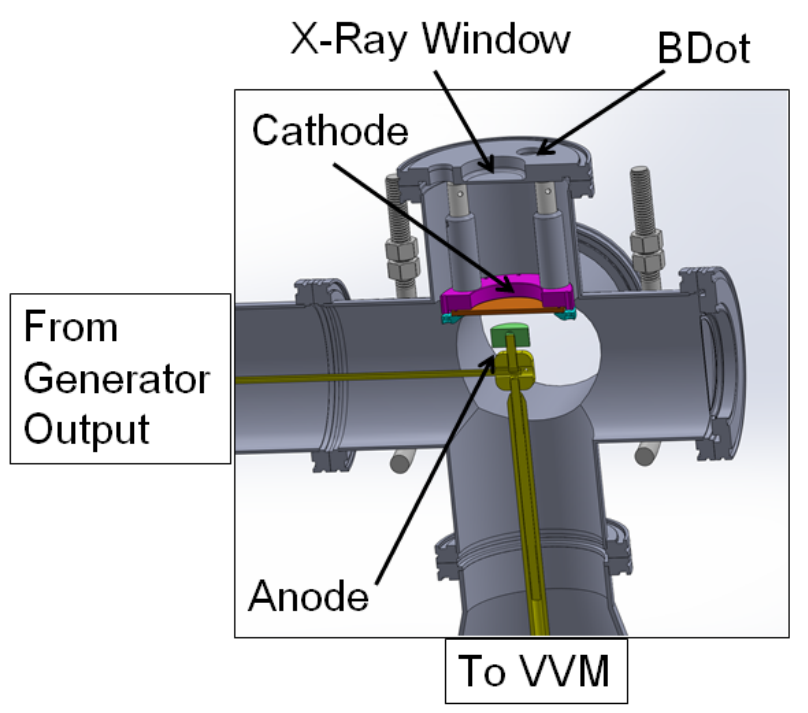

Figure 2. Cross-sectional view of anode and cathode samples inside of an ISO100 six-way cross.

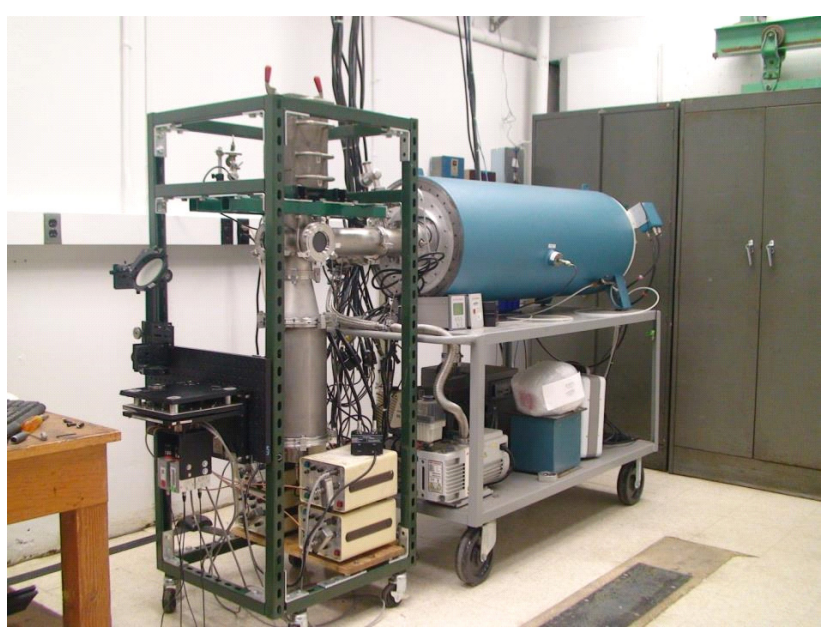

Figure 3. Photograph of the initial experimental setup with the $1 \mathrm{MV}$ generator.

Although the simple geometry of the anode and cathode is very convenient for measuring the onset of explosive cathode emission, there are some caveats regarding the electric field profile on the cathode. The electric field profile on the cathode surface was calculated for various AK gaps using a 2D electrostatic field code. An example 
output plot is shown in Fig. 4. Superimposed on this plot is a rectangle representing a spacer used to set the AK gap. The AK gap can be easily set without touching the cathode surface using precision ground spacer bars as shown. The radial profile of the electric field on the cathode surface is plotted in Fig. 5. With smaller AK gaps the field magnitude is more uniform and drops off rapidly outside of the anode, but is less uniform and drops more gradually with larger AK gaps.

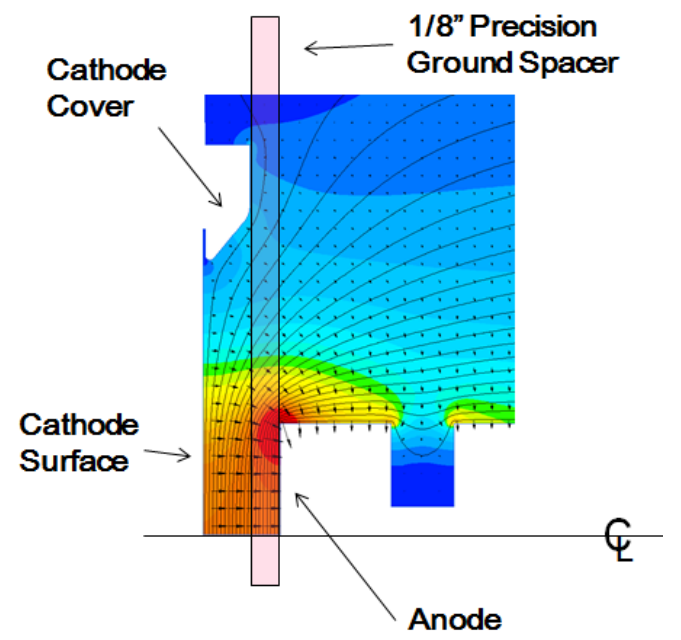

Figure 4. A 2D electric field plot of high field region between anode and cathode (red is highest field, blue lowest field) with spacer used to set AK gap superimposed.

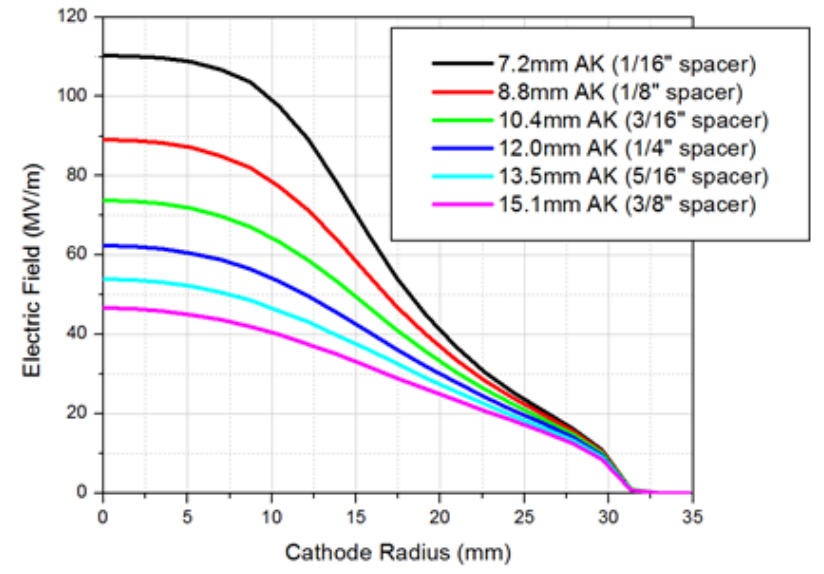

Figure 5. Radial electric field profile along cathode surface with various AK gaps.

Two useful features of this 1-MV generator for these measurements are repeatability and variable output voltage. The output of the generator shows very little shot to shot variation when charged to the same charge voltage as can be seen in Fig. 6a where the output voltages from a seven shot series are overlain. Also, we are able to operate the generator with a charge voltage anywhere between 10 and $35 \mathrm{kV}$. However, there is some change in the output pulse shape, as shown in Fig. 6b. These features allow us to test a cathode sample for the onset of breakdown by increasing the peak applied voltage in small steps until breakdown is observed. The ability to independently adjust output voltage and AK gap over a wide range allows us to check for any voltage dependent effects in the electric field required for breakdown. Note that the peak output voltage of $\sim 800 \mathrm{kV}$ could be increased to $1 \mathrm{MV}$ by increasing the value of the VVM resistance from the current value of $110 \Omega$.
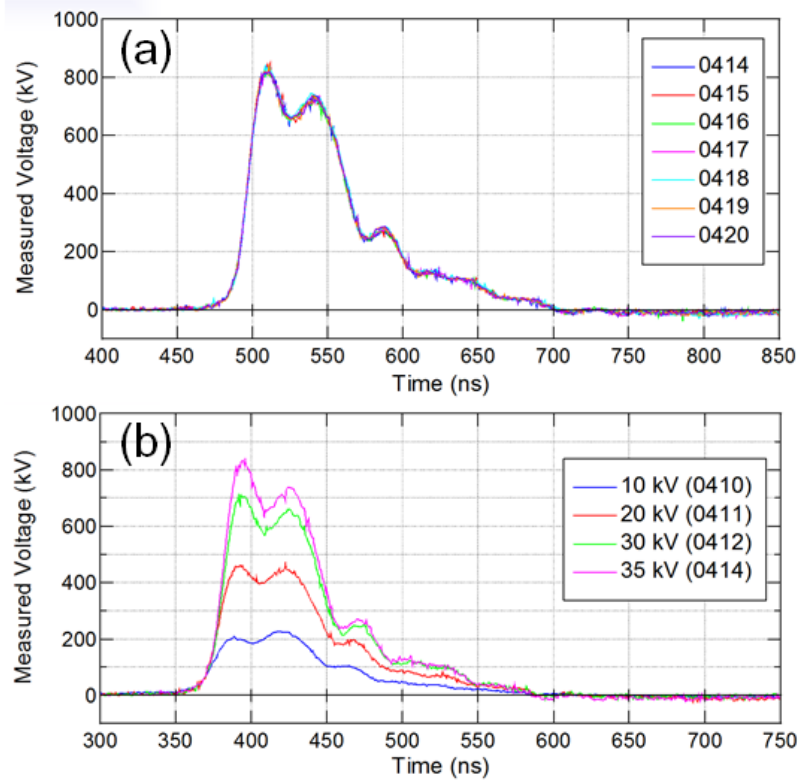

Figure 6. Measured output voltage from (a) a series of seven shots at $35 \mathrm{kV}$ and (b) shots with charge voltage varied between 10 and $35 \mathrm{kV}$.

\section{INITIAL RESULTS}

Our first measurements of cathode emission were with an aluminum cathode and a tungsten anode. Only a handful of samples have so far been tested. Bare aluminum with a 32 finish was chosen for the first tests because it is widely used in pulsed power devices. The cathode was cleaned with acetone, wiped with technical wipes, and then blown with synthetic air. A tungsten alloy was chosen as the anode material for strong x-ray emission and better detection with PIN diode and pinhole camera.

At the time of this writing, only a few samples have been tested. What has been observed so far is very low currents beginning at about $500 \mathrm{kV} / \mathrm{cm}$. The pinhole photos show emission coming mainly from the perimeter of the anode. This emission does not appear to damage the cathode or anode. Repeated shots at $\sim 500 \mathrm{kV} / \mathrm{cm}$ on one sample showed that this current was fairly stable and 
came from varying locations on the anode perimeter. As the voltage is increased, a large current discharge occurs at about $800 \mathrm{kV} / \mathrm{cm}$. This breakdown damages both cathode and anode surfaces. Example pinhole camera photographs using image plates of the anode for both prebreakdown breakdown shots are shown in Fig. 7.
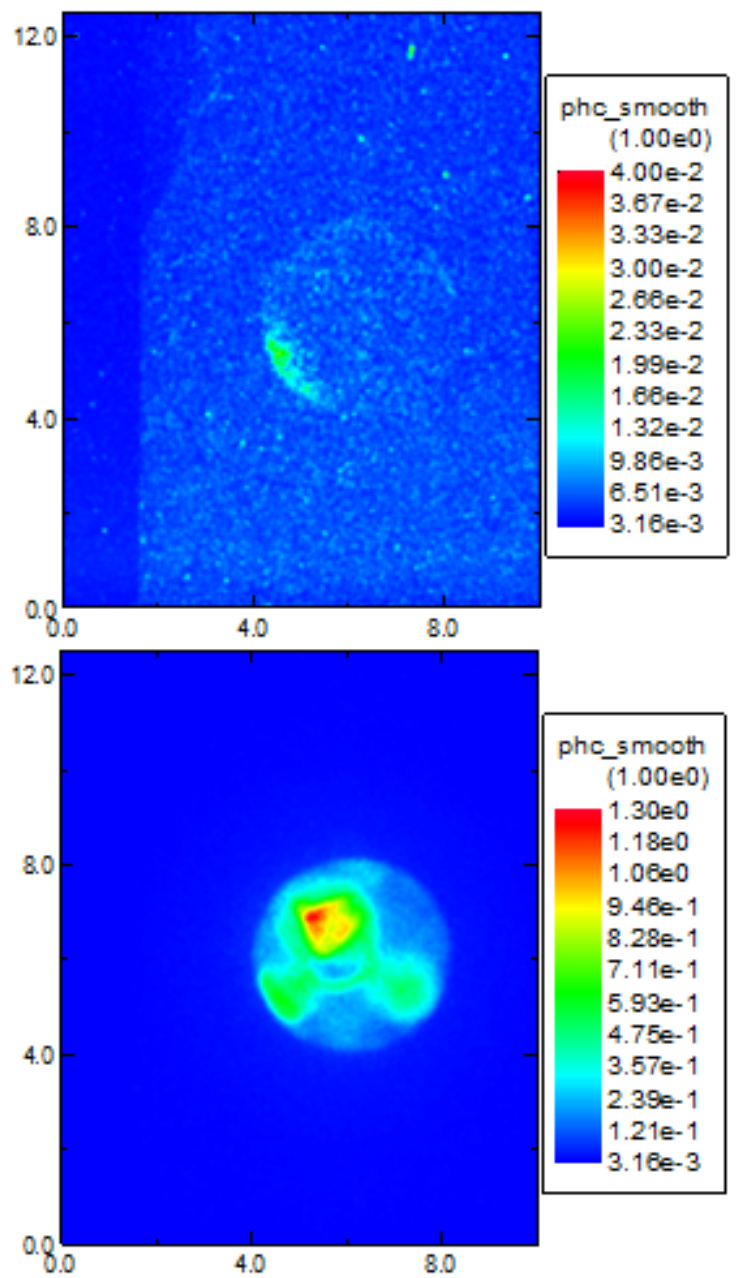

Figure 7. X-ray pinhole camera photographs of x-ray emission from the anode due to both pre-breakdown current (above) and breakdown current (below).

The pinhole camera has proved to be a very useful diagnostic for these experiments. It has helped us localize the pre-breakdown and breakdown current locations. The damaged spots on both anode and cathode matches the bright spots in the breakdown pinhole photo.

Examination of the aluminum cathode damage with SEM shows ridges that are more pronounced that apparent to the naked eye. As shown in Fig. 8, the main damage spot appears as if liquefied metal was pushed out radially from the discharge location and then solidified into a circular ridge. Also, there are small spheres littering the area which may also be solidified liquid aluminum that has attached to the cathode surface.
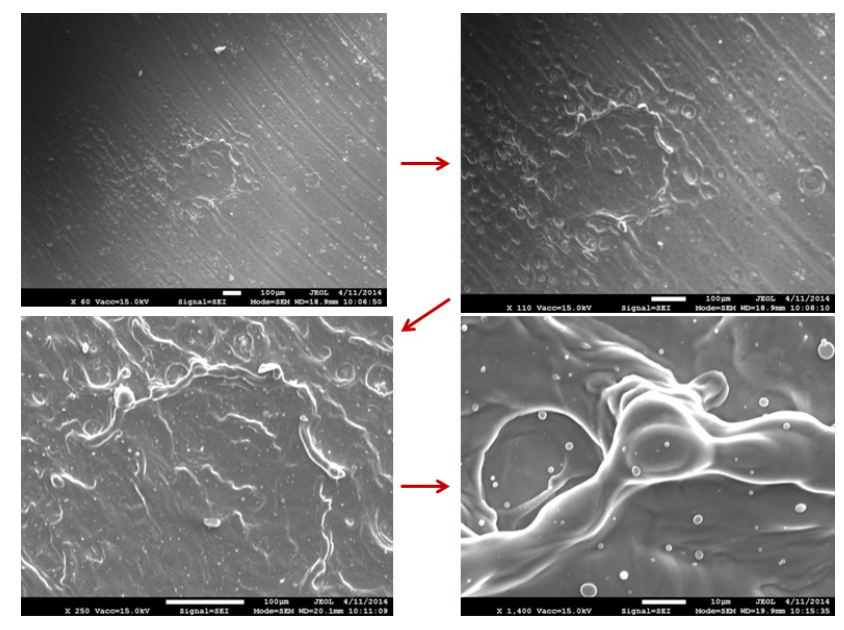

Figure 8. SEM images of the main damaged spot of an aluminum cathode under increasing magnification.

\section{SUMMARY AND FUTURE WORK}

In summary, we have constructed a $1 \mathrm{MV}$ test stand for taking measurement of various forms of particle emission from anode and cathode surfaces with voltage pulses of about $50 \mathrm{~ns}$ in duration. Our initial focus is on explosive electron emission from cathode surfaces. Only a small number of samples have been tested so far.

Soon, we will test cathodes of various metals, finishes, and coatings. Some of the first tests will be with polished aluminum, Aerodag coated aluminum and colloidal silver coated aluminum. SEM images of these surfaces, shown in Fig. 9, make us anxiously await results. Polishing is sometimes done to increase the breakdown strength of a surface. But, at high magnification there are still sharp edges that would be highly field enhanced. Aerodag and colloidal silver are two coatings that are used to produce uniform emission at low fields. Aerodag appears to produce a thin coating that softens sharp edges. Colloidal silver appears to be very smooth at low magnification, but be composed of flakes with protruding sharp edges at high magnification. One goal of this work will be to explain the mechanisms by which these coatings improve uniformity and perhaps find new coatings. Also, we will examine non-emitting cathode coatings and treatments such as anodization of aluminum and coating with thin, insulating films. 


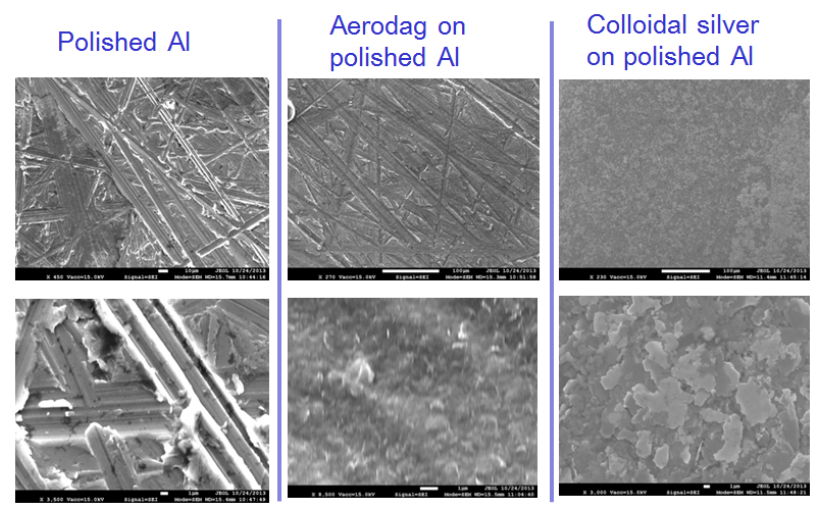

Figure 9. SEM images of polished Al (left), Aerodag coated Al (center), and colloidal silver coated Al (right) with lower magnification above and higher magnification below.

One surprising result thus far is the weak emission, observed at $\sim 500 \mathrm{kV} / \mathrm{cm}$ that is seen at the edge of the anode in pinhole photographs. We now suspect that this is actually due to field desorption of ions from the sharp edge of the anode. These ions must strike the cathode, producing stimulated electron emission from the cathode which then produces x-rays from the anode. When the anode edge was given a $1 / 8$ " radius, the threshold for this emission increased to near the breakdown field. We will repeat these measurements with different anode materials and radii to determine anode effects on cathode emission.

After completing measurements of electron emission from cathode surfaces, we will reconfigure the setup and diagnostics to measure other types of emission. A spectrometer will be used to diagnose anode plasmas. A Thompson parabola will be used to diagnose ion emission from anode and cathode surfaces.

\section{V.REFERENCES}

[1] R. J. Allen, G. Cooperstein, F. C. Young, J. W. Schumer, D. D. Hinshelwood, D. Mosher, D. Holmberg, S. E. Mitchel, "Characterization and optimization of a compact, 1-MV, 6-kA radiography source", 14th International Pulsed Power Conference, June 15-18, 2003, pp. 883-886.

[2] C.S. Athwal and R.V. Latham, Physica 104C, pp.4649,1981

[3] T. W. L. Sanford, J. A. Halbleib, J. W. Poukey, A. L. Pregenzer, R. C. Pate, C. E. Heath, R. Mock, G. A. Mastin, D. C. Ghiglia, T. J. Roemer, P.W. Spence, and G. A. Proulx, J. Appl. Phys. 66, 1989.

[4] R.W. Stinnett and T. Stanley, J.Appl.Physics 53, p.3819, 1982. 